\title{
Editorial
}

\section{Post COVID-19 condition: our understanding and preparedness}

\author{
Md. Titu Miah
}

\begin{abstract}
(c) (i) (2) (2)
Copyright: (C) 202I Miah MT. This is an open access article published under the Creative Commons Attribution-NonCommercial-NoDerivatives 4.0 International License, which permits use, distribution and reproduction in any medium, provided the original work is properly cited, is not changed in any way and it is not used for commercial purposes.
\end{abstract}

Received: 29 May 2021;

Accepted: 15 July 202I

It has been more than a year, Bangladesh is struggling to manage the recurrent waves of SARS coronavirus-2 disease 2019 (COVID-19) along with the rest of the world. While acute management of the crisis is a continuous challenge to the health system, the growing number of patients presenting with various health problems after their recovery from COVID-19 also a great concern. They may experience symptoms long after the acute illness. World Health Organizations (WHO) affirmed the need for recognition, research and rehabilitation of the patients with long symptoms of covid-19 from August 2020. It also established the ICD-10 code for the post-covid-19 condition by September 2020. Although there are no widely accepted definitions of post-COVID-19 condition, a generally agreed definition proposed by the Centers for Disease Control and Prevention (CDC) and accepted by WHO states that 'this condition encompasses a broad range of symptoms (physical and mental) that develop during or after COVID19 , continue for $\geq 4$ weeks, and are not explained by an alternative diagnosis'. ${ }^{1}$ There are many terminologies like, Chronic COVID Syndrome, Late sequelae of COVID-19, Long COVID, Long haul COVID, Long-term COVID-19, Post COVID syndrome, Post-acute COVID-19 and Post-acute sequelae used for this condition by the researchers and health authorities of the world. Finally, WHO and CDC suggested the use of the term 'Post COVID-19 condition' as it is neutral and lacks attribution of causality. ${ }^{2}$

The actual prevalence of the post-covid-19 condition is not presently available due to the lack of robust longitudinal surveillance data. The frequency of long-term symptoms and conditions following SARS-CoV-2 infection varies widely in the literature, ranging from $5 \%$ to $80 \% .{ }^{1}$ One study from

Professor of Medicine and Principal, Dhaka Medical College, Dhaka, Bangladesh.
Bangladesh found the incidence of the post-COVID-19 syndrome was $46 \%{ }^{3}$ Though patients with older age, female gender, severe illness, ICU admission have been reported to develop more post-COVID-19 conditions, younger patients, patients with mild disease including those in good health before SARS-CoV-2 infection have also reported debilitating post-COVID conditions months after acute illness. ${ }^{1}$

The exact pathophysiology of this heterogeneous condition is not established yet. Although efforts are underway to characterize and differentiate the multiple possible etiologies like organ damage resulting from acute phase infection, complications from a persistent hyper-inflammatory state, ongoing viral activity associated with an intra-host viral reservoir, inadequate antibody response and other potential causes, etc. The pattern of onset of post-COVID-19 condition is also heterogeneous. The following patterns may be observed- (i) persistent symptoms and conditions that begin at the time of acute COVID-19 illness; (ii) new-onset late sequelae following asymptomatic disease or a period of acute symptom relief or remission; or (iii) evolution of symptoms and conditions that include some persistent symptoms (e.g., shortness of breath) with the addition of new symptoms or conditions over time (e.g., cognitive difficulties). ${ }^{1}$ Common features of post-COVID-19 conditions so far reported are dyspnea or increased respiratory effort, fatigue, postexertional malaise and/or poor endurance, "Brain fog," cognitive impairment, cough, chest pain, headache, palpitations and/or tachycardia, arthralgia, myalgia, paresthesia, abdominal pain, diarrhea, insomnia and other sleep difficulties, etc. ${ }^{4}$

Post COVID-19 condition involves multiple organ systems of the body. As a result, history varies widely among the patients of Post COVID-19 condition. Evidence from patient experience showed many people feel their symptoms are not 
taken seriously. There are also people who don't realize that their symptoms are connected with COVID-19, so taking time to listen, showing empathy, taking a careful history and making an assessment are important. Comprehensive clinical history should Include- history of suspected or confirmed acute COVID-19, the nature and severity of previous and current symptoms, timing and duration of symptoms since the start of acute infection, history of other conditions. ${ }^{5}$ Thorough physical examination and objective functional assessment is necessary. There is no definite laboratory test that can distinguish post-COVID conditions from other etiologies, in part due to the heterogeneity of post-COVID conditions. A positive SARS-CoV-2 viral test (i.e., reverse transcription polymerase chain reaction [RT-PCR] test or antigen test) or serologic (antibody) test can help assess for current or previous infection; however, these laboratory tests are not required to establish a diagnosis of post-COVID conditions. ${ }^{1}$ Many post-COVID conditions can be improved through already established symptom management medications and non-pharmacological approaches. FDAapproved or over-the-counter medications as well as vitamin or electrolyte supplements may be helpful for indicated illnesses (e.g., headache, anxiety) or documented deficiencies (e.g., vitamin deficiency) after carefully weighing the benefits and risks of pharmaceutical interventions. The following general principles should be kept in mind during treating these patients- 1 . management will usually be pragmatic and symptomatic to optimize function and quality of life, 2. avoidance of over investigation, 3. exclude serious complications and find out possible alternative causes of ongoing symptoms, 4. investigate new or worsening symptoms that could indicate delayed sequelae such as VTE, cardiac complications or pneumonia, 5. consider chest Xray at 12 weeks for those who have had a significant respiratory illness, 6 . where possible, optimize the management of chronic conditions, 7. collaborate with the patient to develop an individualized management plan, 8 . development of multidisciplinary models of care guided by the general practice team. ${ }^{1,6}$

Since August 2020, the Department of Medicine, Dhaka Medical College Hospital first established a post-COVID-19 clinic in this country for the care of the patients discharged from inpatient. Related allied specialties like Pulmonology, Neurology, Psychiatry, Rheumatology, Nephrology, Hematology, Cardiology, Physical Medicine and Rehabilitation, laboratory medicine and social welfare team are working in close collaboration. The clinic has already followed up more than 600 patients. Data from the clinic suggests that approximately $9-12 \%$ of post-COVID patients are readmitted within two months of discharge and nearly $24 \%$ within six months of discharge. Common causes of readmission were breathlessness, respiratory infection, severe weakness, body ache, joint pain, diarrhea, heart problem. Therefore, every COVID-19 dedicated hospital should also establish a post-COVID-19 clinic by their medicine specialists to reduce readmission. Training of the doctors, nurses and other medical staff regarding the management of post-COVID-19 condition should be arranged by the appropriate authority. National guidance for the treatment of the post-COVID-19 condition is also necessary for the consistency and uniformity of treatment nationwide. This will reduce unnecessary treatments. We must develop research protocols to derive data of post-COVID-19 conditions of our own population. Healthcare professionals should be keen and cautious for appropriate diagnosis and management of post-COVID conditions.

\section{References:}

1. CDC. COVID-19. Center for Disease Control and Prevention. [Online] 06 2021. [Cited: 07 24, 2021.] https://www.cdc.gov/ coronavirus/2019-ncov/hcp/clinical-care/post-covidbackground.html.

2. World Health Organization. Expanding our understanding of Post COVID-19 condition [Internet]. 2021. p. 32. Available from:https://www.who.int/publications/i/item/ 9789240025035

3. Mahmud R, Rahman MM, Rassel MA, Monayem FB, Sayeed SKJB, Islam MS, et al. Post-COVID-19 syndrome among symptomatic COVID-19 patients: A prospective cohort study in a tertiary care center of Bangladesh. PLoS One [Internet]. 2021;16(4 April):1-13. Available from: http:/ /dx.doi.org/10.1371/journal.pone.0249644

4. Royal Australia College of General Practitioners. Caring for adult patients with post-COVID-19 conditions. Racgp. 2020;1-9.

5. Sign 161Managing the long-term effects of COVID-19, National guidance for identification, assessment and management, Scottish Intercollegiate Guidelines Network . 2020 .

6. COVID-19: Evaluation and management of adults following acute viral illness. UpTo Date. [Online] [Cited: 07 25, 2021.] https://www.uptodate.com/contents/covid-19-evaluationand management-of adults-following-acute-viral illness? search $=$ long $\% 20$ covid $\% 2019 \&$ source $=$ search_result \& selected Title $=1 \sim 130 \&$ usage_type $=$ default $\&$ display_ rank $=1$. 